Disponible on-line en:

http://revistalogopedia.uclm.es

UNIVERSIDAD DE

CASTIIIA-IA MARCHA

Revista de Investigación en Logopedia 1 (2015) 40-59.

ISSN-2174-5218

\title{
Las pausas en el discurso de individuos con demencia tipo Alzheimer. Estudio de casos
}

\author{
Jhennifer Rodríguez 1, Hernán Martínez 1 y Beatriz Valles 2 \\ 1 Universidad de los Andes, Venezuela \\ 2 Universidad del Zulia, Venezuela.
}

\begin{abstract}
Resumen
Basándonos en el hecho de que la demencia tipo Alzheimer conlleva un deterioro cognitivo que provoca fallas comunicativas y discursivas, el objetivo de esta investigación es analizar el uso y los tipos de pausas que aparecen en el discurso de dos personas que padecen ese tipo de demencia. Los datos que constituyen el corpus de análisis corresponden a muestras de habla realizadas por el Laboratorio de Neurociencias de la Universidad del Zulia. Para el análisis se escogieron al azar aquellos enunciados que contuvieran pausas, tanto silenciosas como sonoras, enfocándonos primordialmente en el parámetro acústico de la duración. Se evidencia que la pausa en el discurso del paciente con demencia tipo Alzheimer es un revelador de déficits de carácter lingüístico y cognitivo. Notamos en los dos discursos analizados el empleo de una considerable cantidad de pausas y períodos más largos de tiempo en la duración de las mismas. Nuestros resultados pueden llegar a ser considerados indicios de que las pausas son fenómenos prosódicos diferenciadores de los discursos de sujetos con demencia, así como posibles parámetros para definir el déficit lingüístico y cognitivo en la enfermedad de Alzheimer.
\end{abstract}

Palabras clave: Alzheimer Discurso Pausas, Prosodia.

The pauses in the speech of individuals with Alzheimer's dementia. Case studies.

\begin{abstract}
Based on the fact that clinical manifestations of Alzheimer's disease show together with communication and discourse problems, the objective of this study is to analyze and classify pauses present in the oral discourse of two individuals having this kind of dementia. A corpus-based study from speech samples collected by Laboratorio de Neurociencias from University of Zulia was made. Voiced and voiceless utterances showing pauses were considered, and duration as the acoustic parameter was particularly studied. Pause showed in the discourse made by patients with Alzheimer's disease reveals linguistic and cognitive impairments. The discourse analyzed from the two speech
\end{abstract}


samples reveals a large number of pauses used in longer periods of time. The results from this study could be considered as early signs that pauses are prosodic elements that show differences in the discourse of patients diagnosed with this type of dementia. Pauses could also be considered as possible parameters for defining linguistic and cognitive impairment in Alzheimer's disease.

Key words: pauses, prosody, speech, Alzheimer.

Correspondencia con los autores: hernan.martinez@ula.ve

Recibido 24 Febrero 2015. Primera revisión 27 Marzo 2015.Aceptado 22 Abil 2015.

\section{Introducción}

La planificación del lenguaje es una actividad cognitiva compleja que requiere el manejo casi simultáneo y en tiempo real de diversos subcomponentes del sistema lingüístico que implican la generación y organización del mensaje, su lexicalización y su ejecución articulatoria (Barkat y Gayraud, 2009). Entre otras cosas, al hablar contamos con un espacio temporal para articular lo que decimos, ordenamos el acto de habla de manera sucesiva para comprender a quien nos habla o para hacer entender a los demás lo que nosotros queremos decir. Los momentos de silencio o vacilación que el hablante emplea en su discurso, sin embargo, se pasan por alto en diversas ocasiones, sin atender al hecho de que estas pausas en el discurso son las que ayudan a la planificación del lenguaje, a que los enunciados sean expresados en secuencias de unidades, capaces de precisar el significado de una expresión y darle sentido.

La pausa lingüística es definida por Mora y Asuaje (2009) como "una interrupción, corte o suspensión del hilo discursivo" (pp. 66) relacionada con mecanismos biológicos, como la toma de aire para respirar, la producción, la percepción y la organización rítmica de los mensajes. Sin embargo, Blondet (2006) opina que la pausa es "cualquier detención de la información, exista o no fonación (...) Una pausa, entonces, no implicará siempre un silencio". Agrega la autora que la pausa se trata de "un parámetro prosódico que interviene en la estructuración del habla debido a que delimita unidades discursivas y, a la vez, semantiza la información” (Blondet, 2006, pp. 57).

Las pausas son clasificadas por Mora y Asuaje (2009) en dos tipos fundamentales. Las silenciosas son "aquellas que corresponden a interrupciones del habla en la producción de un enunciado" (pp. 67). Éstas se dividen en silencios para categorías gramaticales y en silencios con función intencional o estilística, ambos tipos utilizados en discursos retóricos y políticos. En contraparte están las pausas no silenciosas, 
“identificadas como interrupciones momentáneas del fluir discursivo", también conocidas como pausas de hesitación (Blondet, 1999; pp. 22). Se diferencian de las pausas silenciosas por su manifestación a nivel acústico y porque a diferencia de estas últimas la mayoría de las veces las pausas de hesitación no cumplen función gramatical sino que más bien le dan al hablante el tiempo necesario para que lleve a cabo los procesos cognitivos necesarios asociados con la producción del discurso. La agrupación de las pausas de hesitación está definida de la siguiente manera: alargamientos (sonidos percibidos más largos de lo usual), pausas llenas (interrupción del hilo discursivo por alguna emisión sonora, clasificándose a su vez en léxicas, es decir, expresiones que tienen función en la lengua pero cuando se convierten en hesitación se encuentran vacías de significado, como este; y no léxicas, es decir, aquellos sonidos que no poseen significado en el léxico sino en el discurso del idioma, como el alargamiento de a), repeticiones (detenciones del flujo discursivo debido a repeticiones de una unidad y, por último, falsos arranques (detenciones del flujo discursivo pero en este caso son sólo un intento fallido del locutor por iniciar su discurso).

Es importante tener en cuenta que "la duración de la pausa puede variar enormemente, pues es indicadora de una serie de informaciones tanto lingüísticas como extralingüísticas" (Blondet, 2006, pp. 64). Una característica de las pausas es que generalmente no exceden los 3 segundos. Para el español venezolano, Blondet (2006) determina que en el habla normal las pausas lindan entre los 0,4 y 0,7 segundos. Agrega a esto Blondet que todo aquello mayor a los 0.1 segundos es considerado como una pausa gramatical.

\section{Pausas y Alzheimer}

El lenguaje del paciente con demencia tipo Alzheimer deja de ser normal y comienza a ser especial cuando de manera progresiva su memoria se ve afectada, manifestándose en una serie de limitaciones lingüísticas, que se evidencian en una incapacidad para producir textos orales y/o escritos con adecuada coherencia y cohesión, y en la imposibilidad de comprenderlos (Martínez, 2009). Diferentes autores (Sabat, 2001; Valles, 2010; Young, Manthorp y Howells, 2010), señalan que las características que manifiesta el lenguaje de las personas con Alzheimer varían drásticamente de un paciente a otro y de acuerdo con la progresión y etiología de la demencia. Las características más resaltantes son la anomia, la parafasia, el uso de neologismos y de muletillas o palabras carentes de sentido y severas dificultades para seguir el tema de una conversación. Todos estos trastornos provocan una 
comunicación difícil entre los pacientes y sus interlocutores habituales. Desde la fase inicial se manifiestan dificultades para evocar información y en consecuencia seguir de manera eficiente la conversación (Rumbos y Valles, 2010), las fallas en la memoria semántica provocan que la misma se vea limitada, hasta llegar a un grave compromiso lingüístico (Bayles, Tomoeda, Cruz y Mahendra, 2000).

La mayoría de las personas con Alzheimer muestran falta de iniciativa al hablar, lentitud en las respuestas, fluencia interrumpida y coherencia casi inexistente, además de una corrección y repetición de palabras, interpretada por algunos autores "como la incapacidad para redirigir la atención" (Gil,1988:200). Las personas con Alzheimer tienen periodos de mutismo absoluto combinados en ocasiones con logorrea. Se ha hallado que lo más frecuente en esta población es el empobrecimiento de vocabulario y la dificultad de encontrar palabras, por ejemplo, les cuesta más encontrar sustantivos que adjetivos y éstos más que los verbos, siendo las palabras más comunes más fácilmente producidas (Hodges, Salmon y Butters, 1991). Sobre este aspecto, Gil (1988) halla que el declive en la fluencia de palabras no se debe tanto a la pérdida del abastecimiento de palabras sino a un deterioro en el acceso a ellas. Es así, como la mayor parte de los estudios lingüísticos realizados con sujetos con demencia tipo Alzheimer buscan investigar alteraciones en el plano léxico-semántico, principalmente alteraciones en el acceso al léxico (Hodges, Salmon y Butters, 1991).

A estos estudios se agregan otros que describen alteraciones semánticas (Chertkhon y Bub, 1990), sintácticas (Gayraud, Barkat y Lee, 2011) y discursivas tanto en la producción oral como escrita (Obler, Albert y Helm-Estabrooks, 1985). En la bibliografía más reciente sobre el tema también se puede observar una clara preocupación por la identificación precoz de las posibles alteraciones lingüísticas como indicadores para el diagnóstico temprano (Cuetos, Rodríguez-Ferreiro y Menéndez, 2009; Guerrero, 2014).

Desde el punto de vista discursivo, Valles $(2006 ; 2013)$ ha planteado que al entablarse conversaciones con los individuos con demencia tipo Alzheimer se evidencian los problemas que éstos tienen para participar con eficacia en este intercambio, debido a dificultades para entender el contexto y ajustarse a los cambios de turno, variaciones de tema y otros eventos lingüísticos, por lo que la interacción termina siendo muy costosa para ambos interlocutores.

Aso Escario (2005) ha señalado que el discurso del paciente con Alzheimer se presenta fragmentado. Así sostiene que "la enfermedad de Alzheimer puede suponer la 
interrupción del flujo de información de tal manera que la persona que habla tiene dificultad para trasladar sus pensamientos al lenguaje, lo que lleva a una fragmentación de las ideas" (2005, p. 296). Este hecho se evidencia concretamente en el uso y número de las pausas vacías y llenas en el discurso.

Los estudios de la producción del habla en el envejecimiento normal muestran un aumento en la duración de las pausas vacías que pueden ser interpretadas en términos de dificultad de acceso al léxico y enlentecimiento cognitivo. Sin embargo, los estudios sobre las pausas en la producción de los pacientes con Alzheimer han demostrado que ellos producen más pausas de silencio que un adulto mayor sano (Bucks et al., 1999; González, 2000). Tapir-Ladino (2003) estudió las pausas en la producción de pacientes con Alzheimer encontrando que cuando aquellas aparecen en el discurso evidencian la dificultad para recuperar las palabras y verbos además de la dificultad para reformular adecuadamente los mensajes de acuerdo con la situación comunicativa. Este mismo hecho lo afirman para el francés Barkat y Gayraud (2009).

En 1990, Butterworth evaluó las actividades de planificación semántica, léxica y sintáctica en el habla espontánea a través de las pausas. El resultado de tal estudio arrojó que la cadena de habla está organizada en fases de planificación y ejecución en donde las pausas están asociadas a dos procesos: el primero de micro-planificación para la selección léxica ante palabras de baja frecuencia de uso para el hablante, y el segundo de macroplanificación, emparentado con la organización semántica y sintáctica. Por tanto, el proceso de producción del discurso se inicia con la elaboración de una representación general que se va planificando simultáneamente cuando hablamos, por lo que el empleo de pausas se convierte en una estrategia para intentar organizar la cadena de pensamientos.

Por su parte, Blanken, Dittman, Hass y Wallesch (1987) compararon el discurso de personas con Alzheimer, con afasia y sujetos normales. Los autores señalaron que los primeros usaron en su mayoría estrategias para demorar la conversación con el fin de realizar trabajos de búsqueda de palabras, en tanto que los afásicos tendieron a no realizar dichas tareas y a abandonar la conversación.

Al examinar las pausas en el habla, sus funciones dentro de la conversación y la narración en el discurso de cuatro mujeres con Alzheimer de Estados Unidos y Nueva Zelanda, Davis y Maclagan $(2009,2010)$ proponen lo siguiente: 1. las pausas nosilenciosas, alargamientos de sonidos, pausas llenas léxicas y falsos arranques, son usadas para la estructuración del discurso; 2. las pausas silenciosas son usadas durante el acceso 
al léxico, es decir, para encontrar palabras, para planificar una palabra o frase y para compensar pragmáticamente las habilidades de interacción comunicativa que se ven disminuidas en etapas intermedias de la enfermedad de Alzheimer.

En otra investigación, Barkat y Gayraud (2009) tomaron diez pacientes con Alzheimer y diez sujetos sanos, emparejados por edad, sexo y nivel educativo. Se le pidió a cada uno de los participantes que narraran una historia mientras eran grabados. Las pausas silenciosas encontradas fueron codificadas de la siguiente manera: retardo breve (<200 ms.), pausa larga (>200 ms. <1 sg.), interrupción (>1 sg.). Las pausas llenas (alargamiento y repeticiones) también fueron incluidas en el estudio. Se comparó la frecuencia, duración, distribución de las pausas, la relación entre las interrupciones y las estructuras posteriores, y la naturaleza de los elementos que se repiten. Los resultados preliminares mostraron que la proporción en la falta de fluidez fue significativamente mayor en las personas con Alzheimer, afectando el acceso al léxico de los verbos y sustantivos.

Por su parte, Gayraud, Lee y Barkat (2011) estudiaron la relación entre los aspectos verbales y no verbales durante la producción del habla de sujetos con Alzheimer. Se utilizaron las pausas y titubeos como marcadores de la dificultad de planificación y como hipótesis de que la población afectada por el Alzheimer muestra patrones diferentes en el proceso de producción del discurso. Los investigadores compararon la distribución, la duración y la frecuencia de la falta de fluidez del habla en el discurso espontáneo entre veinte personas con Alzheimer y veinte sujetos normales. Se encontró en el discurso de estos sujetos el empleo de una gran cantidad de pausas silenciosas, las cuales ocurren con mayor frecuencia fuera de los límites sintácticos y son seguidos por palabras frecuentes. Además, se evidenció que los sujetos sanos cuando tenían problemas de planificación utilizaban pausas llenas, mientras que los sujetos con Alzheimer no lo hacían.

Basándonos en la hipótesis de que en el discurso de las personas con Alzheimer se pueden apreciar alteraciones cognitivas que se manifiestan mediante el uso de pausas silenciosas y sonoras, este trabajo tiene como objetivo analizar el uso y los tipos de pausas que aparecen en el discurso de dos personas que padecen demencia tipo Alzheimer. Los resultados que podamos suministrar a partir de nuestro estudio serán de mucha importancia para el español ya que para esta lengua no se cuentan con datos relacionados con el uso de pausas en el discurso patológico. 


\section{Método}

\section{Corpus}

El corpus de trabajo está constituido por dos grabaciones audiovisuales realizadas por el Laboratorio de Neurociencias de la Universidad del Zulia perteneciente a la Facultad de Medicina y enmarcado en el proyecto de investigación "Lenguaje y Demencia: un estudio discursivo", desarrollado por Beatriz Valles. Dichas grabaciones se hicieron con dos informantes que padecen demencia tipo Alzheimer a los cuales se les grabó consecutivamente durante un año. En las grabaciones se aprecian interacciones comunicativas, conversaciones en las que participaron las personas con demencia, sus cuidadoras y la propia investigadora. Para la realización de las grabaciones Valles debió visitar los hogares de las familias que aceptaron participar. Estos dos informantes y sus familiares dieron su consentimiento para usar sus datos en el mencionado proyecto.

\section{Muestra}

De ese corpus, es decir, de las dos grabaciones hechas con los dos pacientes con demencia tipo Alzheimer, se procesaron aleatoriamente 11,21 minutos de los cuales 7,21 minutos corresponden a la grabación del Informante 1 y 4 minutos a la del Informante 2. En estos 11,21 minutos analizados se evidencia el uso de pausas en el discurso de los dos informantes. Así, en la muestra se encontraron 63 pausas en total, tanto silenciosas como llenas.

Las duraciones de esas 63 pausas serán comparadas a lo largo del análisis de este trabajo con las proporcionadas por Blondet (2006) para el habla normal.

\section{Informantes}

Un hombre de 80 años y una mujer de 77 años fueron los informantes que participaron en las grabaciones que constituyen el corpus. Ambos informantes son de la ciudad de Maracaibo, Venezuela. El Informante 1 presenta demencia tipo Alzheimer en fase intermedia de tres años de evolución y la Informante 2 la presenta en fase inicial de un año y medio de evolución.

\section{Procesamiento acústico}

Con en el programa Praat fueron procesadas acústicamente las grabaciones. A través del procesamiento se obtuvo el espectrograma de banda ancha con el fin de localizar en el discurso las pausas y su duración. 
Para llevar a cabo el análisis se establecieron ciertas medidas para segmentar las diversas pausas encontradas, guiándonos de los mismos criterios empleados por Blondet (1999) y que explicaremos a continuación.

Para las pausas silenciosas se tomó en cuenta los segmentos en los que no se realizó ningún sonido y que tuvieran una duración considerable (más de 50 ms.).

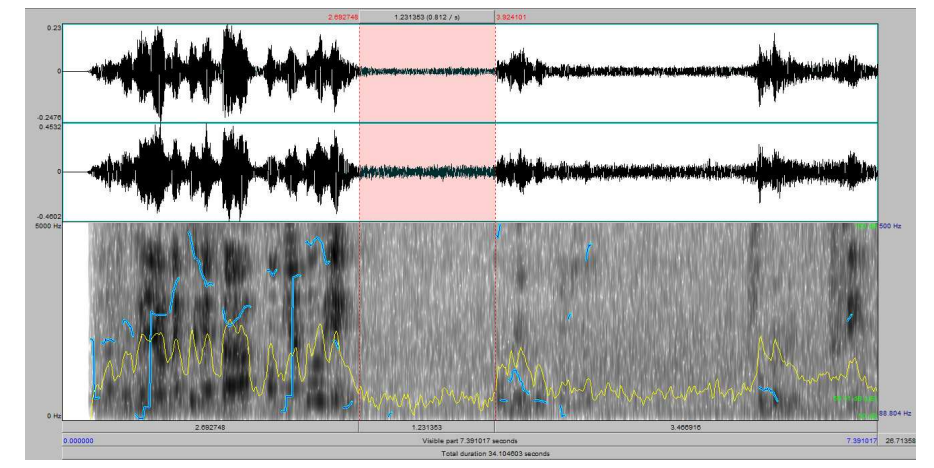

Figura 1. Espectrograma de una pausa silenciosa. Línea 2, T1/Inf. 1/Ev1: “[...] ¿Comiendo? [...] Súper bien”.

Con los alargamientos únicamente se tomó en cuenta la duración del segmento alargado. Las pausas llenas, tanto léxicas como no léxicas, fueron consideradas como una unidad en sí mismas, por lo que al momento de las mediciones se tomaron en cuenta todos los segmentos que la conformaban.

Para los falsos arranques se tomaron en cuenta todos los segmentos que lo conforman. En el caso de las repeticiones también se incluyeron los valores de todas las unidades que conformaban la repetición. Los silencios internos, si se daban, también se tomaron en cuenta.

\section{Resultados}

A continuación, en la Tabla 1, se presenta la distribución de las pausas realizadas por cada uno de los informantes:

Tabla 1: Distribución de las pausas por paciente en el total de la muestra

\begin{tabular}{lccc}
\hline \multicolumn{1}{c}{ TIPO DE PAUSA } & IINFORMANTE $\mathbf{1}$ & INFORMANTE 2 & TOTAL \\
Pausas silenciosas & 28 & 8 & 36 \\
Pausas llenas no léxicas alargadas & 1 & 0 & 1 \\
Pausas llenas léxicas no alargadas & 0 & 3 & 3 \\
Falsos arranques & 2 & 1 & 3 \\
Repeticiones & 10 & 5 & 14 \\
Alargamientos & 3 & 0 & 3 \\
\hline TOTAL & $\mathbf{4 5}$ & $\mathbf{1 7}$ & $\mathbf{6 3}$
\end{tabular}


A simple vista es posible notar la diferencia de realizaciones pausales entre uno y otro informante. Además, es bastante considerable la cantidad de pausas encontradas en tan solo 11,21 minutos de grabación (63 pausas).

Las pausas más empleadas por ambos informantes fueron las pausas silenciosas, seguidas de las repeticiones. Se dio el caso en el que ciertas hesitaciones encontradas no fueron producidas por alguno de las informantes, como sucedió con las pausas llenas no léxicas y alargamientos que no las utilizó la Informante 2, así como las pausas llenas léxicas que no fueron empleadas por el Informante 1.

\section{Pausas silenciosas}

Las pausas silenciosas fueron las más empleadas durante el discurso de ambos informantes. En el discurso del Informante 1 aparecieron 28 y 8 en el de la Informante 2.

Como se mencionó anteriormente, Blondet (2006) sostiene que las pausas silenciosas tienen una duración mayor a los $0.5 \mathrm{seg}$. y menor a los $3 \mathrm{seg}$. en la producción de un sujeto normal, por lo que es importante hacer notar que el Informante 1 en siete ocasiones supera este promedio, incluso llega a emplear una pausa de $7 \mathrm{seg}$. Por su parte, en el discurso de la Informante 2 la duración de este tipo de pausas entraría dentro de los valores normales, sólo en una ocasión esta Informante emplea una pausa mayor a lo establecido como normal (de 5,9 seg.).

Además, hemos podido notar que el Informante 1, en la medida en que va avanzando su discurso, va realizando con mayor frecuencia y duración las pausas silenciosas.

Tabla 2: Promedio de la duración de pausas silenciosas.

\section{Informante 1}

Duración total de las pausas: $65,21 \mathrm{seg}$. Total de pausas: 28

Resultado: $65,21 / 28=2,32$ seg.

\section{Informante 2}

Duración total de las pausas: $15,88 \mathrm{seg}$. Total de pausas: 8 Resultado: $15,88 / 8=1,98$ seg.

En la Tabla 2, observamos el promedio de la duración de las pausas silenciosas producidas por cada uno de los informantes. Así, se obtuvo una media pausal de 2,32 seg. para el Informante 1 y de 1,98 seg. para la Informante 2. Estos valores promedio se corresponderían con los dados para la duración de las pausas silenciosas producidas por 
sujetos normales. Sin embargo, es preciso señalar que, sobre todo el Informante 1, en algunas ocasiones usa pausas con duraciones mayores a los $3 \mathrm{seg}$.

Este tipo de pausas, con duración mayor a los 3 seg., aparecen en el discurso del Informante 1 durante el proceso de acceso al léxico, durante la búsqueda de la(s) palabra(s) deseada(s), algunas veces sin éxito. Veamos el siguiente ejemplo:

(a) Cuidador: Estamos en diciembre, ella te pregunta ¿qué vas a hacer en diciembre?

Informante 1: Nosotros aquí, yo por ejemplo [...] a veces que salgo un poquito hasta ahí, hasta el [...] ya, más nada.

En (b) se puede apreciar que este tipo de pausas en el discurso de la Informante 2 también es usado durante el proceso de acceso al léxico:

(b) Entrevistadora.: ¿Y qué le puso el doctor Carlos?

Informante 2: [...] ¿Qué me puso? [...] No, yo lo que iba era a

Cuidadora: ¿Qué medicina te ponían?

Informante 2: [...] Yo no me acuerdo [...] tengo tiempo que no voy.

\section{Pausas llenas no léxicas alargadas}

Como se muestra en la Tabla 3 las pausas llenas no léxicas sólo las empleó el Informante 1, en una sola ocasión, haciéndola a su vez alargada y utilizando la vocal [u:]. En el discurso de la Informante 2 no se encontró este tipo de pausas.

Tabla 3: Promedio de la duración de pausas llenas no léxicas alargadas.

\section{Informante 1}

Duración total de las pausas: 2,69 seg.

Total de pausas: 1

Resultado: 2,69 / 1 = 2,69 seg.

\section{Informante 2}

Duración total de las pausas: $0 \mathrm{sg}$.

Total de pausas: 0

Resultado: $0 / 0=0$ seg.

Por su parte, en la Tabla 3 se presenta el promedio de duración de este tipo de pausas, que sólo se obtuvo del Informante 1, fue de 2,69 seg., rayando casi en el límite de lo establecido como una pausa silenciosa normal. 
Este tipo de pausa apareció durante la producción del siguiente fragmento discursivo:

(a) Entrevistadora: ¿Usted sale a caminar con la Sra. L?

Informante 1: [u:] a donde quiera que sea.

\section{Pausas llenas léxicas no alargadas}

Las pausas llenas léxicas no alargadas también fueron realizadas por un solo informante, tal como se muestra en la Tabla 4. La Informante 2 realizó este tipo de hesitación en tres ocasiones, empleando la palabra este, con una duración promedio de 0,52 seg. en los tres momentos en que la utilizó.

Tabla 4: Promedio de la duración de pausas llenas léxicas no alargadas.

Informante 1 Informante 2

Duración total de las pausas: $0 \mathrm{seg}$. Duración total de las pausas: 1,58 seg. Total de pausas: $0 \quad$ Total de pausas: 3

Resultado: $0 / 0=0 \mathrm{seg}$. Resultado: $1,58 / 3=0,52 \mathrm{seg}$.

Este tipo de pausa apareció durante la producción de los siguientes fragmentos discursivos:

(a) Informante 2: Este, voy así cuando voy es porque me estoy bebiendo [...] el cal, el, una de calcio que me pusieron.

(b) Informante 2: Pero a nosotros, este, a nosotros nos llevan y nos dejan allá, entonces como no sabemos la hora nos venimos caminando desde la placita ella y yo.

(c) Entrevistadora: Entonces, ¿cuándo comienzan las ferias?

Informante 2: Este, como dos días antes.

Este tipo de pausas evidencian que la Informante 2 está planificando, estructurando lo que va a decir. Sirven estas pausas de hesitación para ganar tiempo durante la producción discursiva. 


\section{Falsos arranques}

Durante la producción discursiva de los dos informantes se pudieron apreciar falsos arranques. El Informante 1 los empleó en dos ocasiones: en (a) el falso arranque tuvo una duración de 1,2 seg. y en (b) tuvo una duración de 0,65 seg.

(a) Entrevistadora: ¿Cuántos años tiene el Sr. N., Sra. L?

Cuidador: Ochenta cumplió el seis de este mes.

Informante 1: Yo creía que era más con... más pequeño.

(b) Cuidador: Ellos me están preguntando quiénes son los que vivimos aquí, cuántas personas habemos.

Informante 1: Hay veces que... Eso sí es verdad que hay veces que no, no [...] y eso no es nada de: nada sino que a veces hay veces que ni sé quién soy yo.

Por su parte, la Informante 2 sólo utilizó este tipo de hesitación una vez, llevándola a cabo en $0,88 \mathrm{seg}$.

(c) Entrevistadora: ¿Y no está tomando nada?

Informante 2: [Gesto negativo] Este, voy así cuando voy es porque me estoy bebiendo [...] el cal, el, una de calcio que me pusieron.

El cálculo del promedio de duración de este tipo de pausas fue de 0,92 seg. para el Informante 1 y de $0,88 \mathrm{seg}$. para la Informante 2, evidenciándose una mínima diferencia entre ambos de 0,4 seg (cf. Tabla 5).

Tabla 5: Promedio de la duración de falsos arranques.

\section{Informante 1}

Duración total de las pausas: $1,85 \mathrm{seg}$. Total de pausas: 2

Resultado: $1,85 / 2=0,92 \mathrm{seg}$.

\section{Informante 2}

Duración total de las pausas: $0,88 \mathrm{sg}$.

Total de pausas: 1

Resultado: $0,88 / 1=0,88$ seg.

Los falsos arranques que se pueden apreciar en el discurso de los dos informantes representan intentos fallidos por iniciar su discurso ( $a, b$ y c). 


\section{Repeticiones}

En lo que se refiere a las repeticiones, el Informante 1 es quien, también, produjo un mayor número de este tipo de pausas. En el discurso del Informante 1 se encontraron las siguientes repeticiones:

(a) Entrevistadora: ¿Está tomando alguna medicina?

Informante 1: [...] Ella sabe, yo sé [...] Y estoy [...] bien, bien, bien porque [...] yo no tengo nada de malo, por aquí, por allá, por allá.

(b) Entrevistadora: ¿Y su nieta? ¿Cómo se llama su nieta? Su última nieta.

Informante 1: [...][Mira a la cuidadora pidiendo ayuda]

Cuidador: La bebecita de aquí, la bebecita.

Informante $1: ; A h$ ! Eso es lo malo que he tenido últimamente, que a veces no me acuerdo de muchas cosas, esos, esos, esos son los años.

(c) Entrevistadora: ¿Y se acuerda en qué lugar de Maracaibo nació?

Informante 1: [...] Esas, esas son las cosas que tiene N [dice su nombre completo] que hay veces que ni me acuerdo de algunas cosas, eso es lo mío [...] no soy como esa gente que sale a la calle taka, taka, taka, ¡hombre! Taka, taka, taka; nada de eso.

En la mayoría de los casos de repeticiones encontradas se observó triplicaciones y en el resto duplicaciones. Sólo en (d) se evidenció una duración bastante larga de este tipo de pausa: 3,05 seg., diferenciándose temporalmente del resto de repeticiones encontradas en el discurso de este informante.

(d) Entrevistadora: ¿Pero está comiendo bien?

Cuidador: Sí, si supieras que sí... Y en la tarde también que si arepita, que si pan otra vez con... a veces que le hago maicena, le hago fresca chicha, algo pues más sano... plátano cocido...

Informante 1: Lo mejor que tengo yo es que yo [...] nada $d e, d e[\ldots] d e$ psss, ¡hey! Dame dos a mi [gesto de comer].

Por su parte, la Informante 2 realizó varias duplicaciones (e), una triplicación (f) y una cuadruplicación $(\mathrm{g})$ : 
(e) Entrevistadora: A lo mejor le toca ir caminando o por lo menos se regresa, pues, caminando. Qué bueno, eso está bien bueno, no deje de ir a votar.

Informante 2:¡Ajá! Hasta, hasta con las muletas voy.

(f) Informante 2: A una, a una, a una prima de nosotros la operaron de las rodillas y se cayó y se mató.

(g) Entrevistadora: ¿Quién le dijo que no barriera?

Informante 2: $\boldsymbol{E l}, \boldsymbol{e l}, \boldsymbol{e l}, \boldsymbol{e l}$ traumatólogo que me vio.

Al calcular el promedio de duración de las repeticiones se obtuvo que para ambos informantes la media es bastante similar: 1,43 seg. para el Informante 1 y 1,46 seg. para la Informante 2, a pesar de que el número de hesitaciones de este tipo fue el doble en el Informante 1 (cf. Tabla 6).

Tabla 6: Promedio de la duración de las repeticiones.

\section{Informante $1 \quad$ Informante 2}

Duración total de las pausas: 14,34 seg. $\quad$ Duración total de las pausas: 7,34 seg.

Total de pausas: $10 \quad$ Total de pausas: 5

Resultado: $14,34 / 10=1,43 \mathrm{seg} . \quad$ Resultado: 7,34 / $5=1,46 \mathrm{seg}$.

\section{Alargamientos}

Durante el discurso del Informante 1 se encontraron dos alargamientos:

(a) Cuidador: Ellos me están preguntando quiénes son los que vivimos aquí, cuántas personas habemos.

Informante 1: Hay veces que... Eso sí es verdad que hay veces que no, no [...] y eso no es nada de... nada sino que a veces hay veces que ni sé quién soy yo.

(b) Entrevistadora: ¿Y no le gusta la pelota? ¿No le gusta el juego de béisbol? Informante 1: Sí me gusta, lo que pasa es que muchas veces uno no puede ir tan, tan, $\tan [\ldots]$ pero todas esas cositas a mí me gustan $[\ldots]$ Yo soy una persona que, que me gusta todo lo bueno $\mathbf{y} \ldots[. .$.$] tranquilito (casilla 2).$

La Informante 2 no empleó este tipo de hesitación. El promedio de duración de los alargamientos producidos por el Informante 1 fue de $0,71 \mathrm{seg}$. 
Tabla 7: Promedio de la duración de los alargamientos.

\section{Informante 1}

Duración total de las pausas: $1,42 \mathrm{seg}$.

Total de pausas: 2

Resultado: $1,42 / 2=0,71 \mathrm{seg}$.

\section{Informante 2}

Duración total de las pausas: $0 \mathrm{sg}$.

Total de pausas: 0

Resultado: $0 / 0=0$ seg.

En la Figura 2 se presenta el promedio de duración pausal total, es decir, el promedio de duración de todos los tipos de pausas encontrados en el discurso de los dos pacientes con demencia tipo Alzheimer.

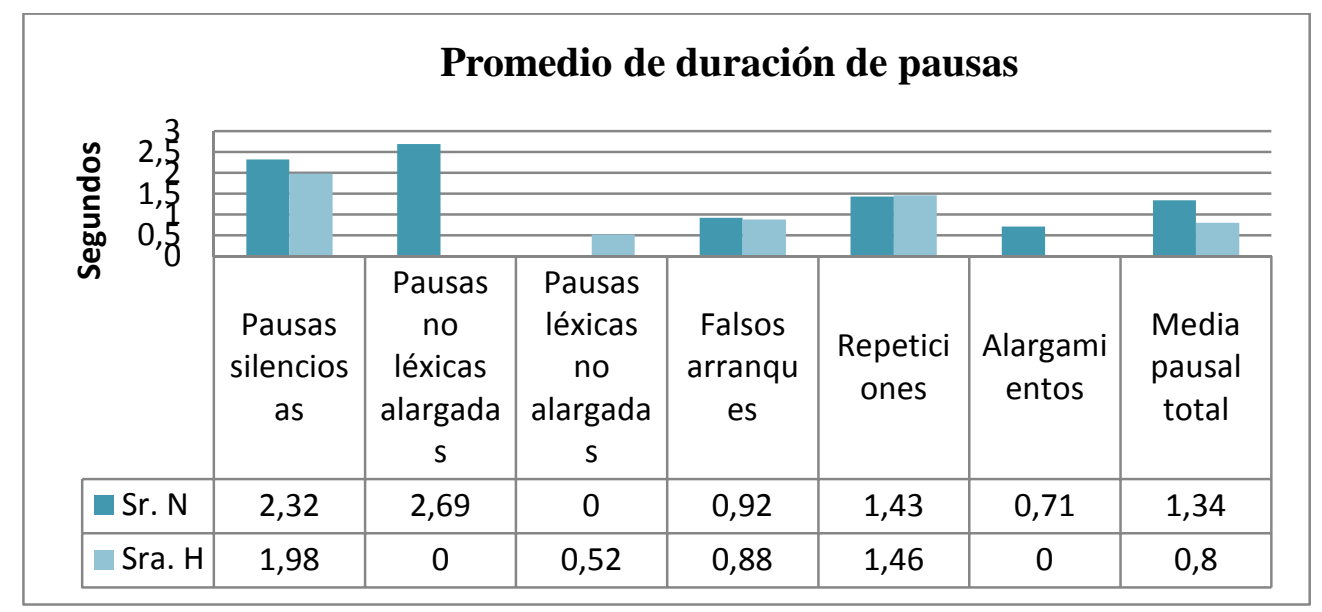

Figura 2: Promedio de duración total de pausas.

En las casillas de la primera fila se encuentran el tipo de pausa y al final la media pausal total; en las casillas de la segunda fila el promedio de duración de todas las pausas empleadas por el Informante 1 y en las casillas de la tercera fila el promedio de duración de las pausas empleadas por la Informante 2. Una vez analizados los datos entre los sujetos con demencia tipo Alzheimer podemos evidenciar que durante sus discursos el Informante 1 usó 1,34 seg. para pausas y que la Informante 2 usó 0,80 seg. Ahora bien, para poder constatar si estos datos son idiosincrásicos de este tipo de sujetos o si, por el contrario, pueden ser similares a algunos de los resultados obtenidos en el análisis del discurso de hablantes normales durante una entrevista, en la siguiente tabla se compara la duración de los distintos tipos de pausas usados por sujetos normales y por nuestros informantes durante la entrevista: 
Tabla 8: Tabla comparativa entre los distintos tipos de pausas usados por sujetos normales y por nuestros informantes

\begin{tabular}{clc}
\hline $\begin{array}{c}\text { Promedio de duración de pausas en un } \\
\text { sujeto normal }\end{array}$ & $\begin{array}{l}\text { Promedio de duración de pausas en un } \\
\text { sujeto con demencia tipo Alzheimer }\end{array}$ \\
Media entrevista & $0,61 \mathrm{seg}$. & Media Inf. 1 $1,34 \mathrm{seg}$. \\
& Media Inf. $2 \quad 0,80 \mathrm{seg}$. \\
\hline
\end{tabular}

Como puede observarse, claramente la diferencia en la duración de pausas entre los dos tipos de hablantes se encuentra marcada por la distancia temporal de casi un segundo de diferencia entre ellos. De este hecho suponemos, junto con otros investigadores citados anteriormente, que el discurso de los sujetos que padecen Alzheimer es más pausado debido a las grandes dificultades para encontrar formas lingüísticas concretas (palabras, verbos, etc.) que sirvan de recipiente para sus ideas, lo que condiciona su discurso haciéndolo más lento y menos fluido que el de los sujetos normales.

\section{Conclusiones}

El análisis de los datos arrojó una muestra total de 63 pausas silenciosas y sonoras contenidas en los discursos de los dos informantes estudiados. El Informante 1 empleó una mayor cantidad de pausas (45 en total): 28 pausas silenciosas (con un promedio de 2,32 seg.), 1 pausa llena no léxica (2,69 seg.), 2 falsos arranques (0,92 seg.), 10 repeticiones $(1,43 \mathrm{seg}$.) y 3 alargamientos $(0,71 \mathrm{seg}$.), obteniendo una media pausal total de 1,34 seg.; mientras que la Informante 2 realizó 17 pausas en total: 8 pausas silenciosas (con un promedio de 1,98 seg.), 3 pausas llenas léxicas (0,52 seg.), 1 falso arranque (0,88 seg.) y 5 repeticiones (1,46 seg.), con un promedio total de $0,80 \mathrm{seg}$. en el empleo de pausas.

Un primer acercamiento al estudio de las pausas en el discurso de sujetos con demencia tipo Alzheimer evidencia que estos individuos experimentan un mayor esfuerzo cognitivo a la hora de planificar su producción lingüística. El uso y la duración de las pausas encontradas en los discursos de los informantes sugieren, como lo han señalado otros investigadores (Tapir-Ladino, 2003; Barkat y Gayraud, 2009; Gayraud, Lee y Barkat, 2011), que tienen dificultades para acceder al léxico almacenado en sus memorias, dificultad para encontrar las palabras que le permitirían expresarse durante la comunicación. 
Además, hemos podido notar que los sujetos con Alzheimer que han servido de informantes para este trabajo no son capaces de mantener intacto el hilo discursivo (cf. Valles, 2013), dispersándose en algunas ocasiones del tema concreto de su intervención en la conversación o simplemente no pudiendo terminar la idea que desean plantear. A esto se suma el uso de correcciones durante el discurso, como se observa en las hesitaciones estudiadas, que hacen pensar en que existe un auto-monitoreo del discurso, es decir, las correcciones son una muestra de que estos informantes son capaces aún de identificar las fallas en su expresión.

A través del análisis pudimos ver que la Informante 2, quien presentaba una demencia en fase inicial al momento de recolección de las grabaciones, hace uso menos frecuente de los distintos tipos de pausas analizados; además, pudimos apreciar que la duración de las pausas utilizadas por esta hablante se corresponde con los parámetros establecidos como normales para el español por Blondet (2006). Este hecho nos permite sugerir que los procesos de planificación y producción del habla en esta informante se han visto poco afectados. Por su parte, en el discurso del Informante 1, que se hallaba en la fase intermedia de la enfermedad, se evidencia a través del uso y la cantidad de las pausas cómo comienza a haber dificultades de memoria, dificultades en el proceso de producción del habla debidas a fallas en el acceso al componente léxico-semántico. Su discurso fue menos fluido, tuvo mayor uso de hesitaciones, la lentitud para responder a las preguntas hechas por la entrevistadora fue muy notoria, con gran empleo de pausas iniciales.

Todo lo planteado anteriormente induce a pensar que el discurso de los sujetos estudiados es pausado. Este hecho se puede apreciar durante los momentos en los que los hablantes planifican lo que van a decir; aunque algunas veces no lo logren con éxito, rompiendo, como plantea Cabedo (2009), con "la linealidad prosódico-comunicativa". En el caso de los sujetos con demencia tipo Alzheimer, el uso de las pausas durante su discurso es mayor que el que podría presentar un hablante sin trastornos lingüísticos lo cual afecta proporcionalmente el intercambio comunicativo.

De este modo, al igual que otros investigadores (Tapir-Ladino, 2003; Barkat y Gayraud, 2009; Gayraud, Lee y Barkat, 2011) proponemos que nuestros resultados pueden llegar a ser considerados indicios de que las pausas son fenómenos prosódicos diferenciadores de los discursos de sujetos normales y de aquellos portadores de la demencia, así como posibles parámetros para definir el déficit lingüístico y cognitivo en 
la enfermedad de Alzheimer, a pesar de que las limitaciones del análisis estuvieron dadas por una muestra pequeña, tratándose de un estudio de casos.

\section{Referencias}

Aso, J. (2005). Demencia: investigación, diagnóstico y tratamiento. Barcelona: Masson. Barkat, M., y Gayraud, F. (2009). Pauses et hésitations dans le discours de patients Alzheimer et chez la personne âgée saine. 3e Journées de Phonétique Clinique.

Bayles, K., Tomoeda, C., Cruz, R., y Mahendra, N. (2000). Communication abilities of individuals with late-stage Alzheimer disease. Alzheimer Disease and Associated Disorders, 14, 176-181.

Blanken, G., Dittman, J., Hass, J., y Wallesch, C. (1987). Spontaneous speech in senile dementia and aphasia. Implications for a neurolinguistic model of language production. Cognition, 27, 247-274.

Blondet, M. (1999). Estudio acústico-prosódico de los fenómenos sonoros de hesitación: análisis contrastivo entre los dialectos andino y central. Trabajo de Grado de Maestría. Mérida: Universidad de Los Andes.

Blondet, M. (2006). Variaciones de la velocidad de habla en español: patrones fonéticos y estrategias fonológicas. Un estudio desde la producción. Tesis doctoral. Mérida: Universidad de Los Andes.

Butterworth B. (1990). Aportaciones del estudio de las pausas en el habla. En F.Valle (Ed.), Lecturas de psicolingüística. Comprensión y producción del lenguaje. Madrid: Alianza Editorial.

Cabedo, A. (2009). Sobre algunos factores prosódicos en pacientes con afasia de Wernicke. Phonica: Habla, Voz y Sonido, 5, 5-20.

Chertkhon, H., y Bub, D. (1990). Semantic memory loss in Alzheimer's type: what do various measures measure? Brain, 113, 397-417.

Cuetos, F., Rodríguez-Ferreiro, J., y Menéndez, M. (2009). Semantic markers in the diagn osis of neurodegenerative dementias. Dementia and Geriatric Disorders, $28,267-274$.

Davis, B., y Maclagan, M. (2009). Examining pauses in Alzheimer's discourse. American Journal of Alzheimer's Disease and Other Dementias, 24, 141-154. 
Davis, B., y Maclagan, M. (2010). Pauses, fillers, placeholders and formulaicity in Alzheimer's discourse: Gluing relationships as impairment increases. En N. Amiridze, B. Davis y M. Maclagan (Eds.), Fillers, pauses and placeholders. (pp. 118-179). Philadelphia: John Benjamins Publishing Company.

Gayraud, F., Lee, H., y Barkat, M. (2011). Syntactic and lexical context of pauses and hesitations in the discourse of Alzheimer patients and healthy elderly subjects. Journal of Clinical Linguistics and Phonetics, 25, 198-209.

Gil, D. (1988). El lenguaje en la enfermedad de Alzheimer. Revista de Logopedia, Foniatría y Audiología, 8, 199-205.

Guerrero, Y. (2014). Is semantic impairment a predictive sign for Alzheimer disease? Revista de Logopedia, Foniatría y Audiología. 34, 137-43.

Hodges, J., Salmon, D. y Butters, N. (1991). The nature of naming deficits in Alzheimer's and Huntingdon's disease. Brain. 114, 1547-1558.

Martínez, A. (2009). El Alzheimer. Madrid: CSIC.

Mora, E., y Asuaje, R. (2009). El canto de la palabra: una iniciación al estudio de la prosodia. Mérida: Universidad de Los Andes.

Obler, L., Albert, M., y Helm-Estabrooks, N. (1985). Empty speech in Alzheimer's disease and fluent aphasia. Journal of Speech Hearing Reserch, 28, 405-410.

Rumbos, H., y Valles, B. (2010). Análisis discursivo de los textos narrativos en el adulto mayor con queja de memoria. En B. Gallardo-Paúls y V. Moreno (Eds.), Estudios de Lingüística Clínica: Aplicaciones Clínicas. Valencia: Publicaciones de la Universitat de Valencia.

Sabat, S. (2001). The experience of Alzheimer's disease. Life through a tangled veil. Massachusetts: Blackwell Publishers Inc.

Tapir-Ladino, M. (2003). Análisis de los enunciados producidos después de una pausa en la conversación de enfermos de Alzheimer y de senescentes. Revista de Lingüística Teórica y Aplicada, 41, 103-118.

Valles, B. (2006). La cohesión y la coherencia en la conversación del paciente con demencia: un estudio discursivo. En B. Gallardo, C. Hernández y V. Marrero. (Eds.), Lingüística clínica y neuropsicología cognitiva. Actas del primer congreso nacional de lingüística clínica (pp. 180-191). Madrid: UNED.

Valles, B. (2010). La atención del lenguaje y la comunicación en las personas con demencia tipo Alzheimer. En Alzheimer: no estás solo. Caracas: Fundación Alzheimer de Venezuela-Capítulo Nueva Esparta. 
Valles, B. (2013). Una aproximación al estudio de la conversación de la persona con Alzheimer y sus interlocutores sanos. Revista de Investigación en Logopedia. 2, 96-119.

Young, M., y Howells, C. (2010). Comunicación y demencia. Nuevas perspectivas. Barcelona: Editorial UOC. 